\title{
Discovery of Ambiguous Patterns in Sequences Application to Bioinformatics
}

\author{
Gerard Ramstein ${ }^{1}$, Pascal Bunelle ${ }^{1}$, and Yannick Jacques ${ }^{2}$ \\ 1 IRIN, Ecole polytechnique universitaire de Nantes, \\ Rue Christian Pauc, La Chantrerie, BP 60602, F-44036 Nantes Cedex 3, France \\ gramstei@ireste.fr \\ 2 INSERM U.463, Institut de Biologie, \\ 9 Quai Moncousu, F-44035 Nantes Cedex 1, France \\ yjacques@nantes.inserm.fr
}

\begin{abstract}
An important issue in data mining concerns the discovery of patterns presenting a user-specified minimum support. We generalize this problematics by introducing the concept of ambiguous event. An ambiguous event can be substituated for another without modifying the substance of the concerned pattern. For instance, in molecular biology, researchers attempt to identify conserved patterns in a family of proteins for which they know that they have evolved from a common ancestor. Such patterns are flexible in the sense that some residues may have been substituated for others during evolution. $\mathrm{A}[\mathrm{B} \mathrm{C}]$ is an example of notation of an ambiguous pattern representing the event $\mathrm{A}$, followed by either the event B or C. A new scoring scheme is proposed for the computation of the frequency of ambiguous patterns, based on substitution matrices. A substitution matrix expresses the probability of the replacement of an event by another. We propose to adapt the Winepi algorithm [1] to ambiguous events. Finally, we give an application to the discovery of conserved patterns in a particular family of proteins, the cytokine receptors.
\end{abstract}

\section{Introduction}

Data mining techniques focus on the discovery of regularities underlying large collections of data and reveals new insight into the hidden organisation of these raw data. One important problem of data mining thus concerns the search of frequent patterns in sequential data, i.e. collections of sequences of events. We propose in this paper an extension of the Winepi algorithm [1] for the discovery of ambiguous patterns. Such patterns can be encountered in many situations. For example in insurance, a cardio-vascular history may present various forms such as arterial hypertension, infarction, phlebistic, etc. All these forms do not have the same gravity degree and therefore should be differently weighted. An interest of our method is indeed that it permits to characterize the representativeness of an event. In supermarket basket data, the purchase of a printer can be differently evaluated according to the power of the device or its characteristics (e.g laser or inck jet printer). Note that the concept of ambiguous event 
encompasses the notion of taxinomy, but is more flexible ; we do not impose that ambiguous events must belong to a same class.

We call pattern a sequence of events that frequently occur in the observed database. Generally a pattern is constrained to belong to a sliding window that limits the search space. A window of size $w$ can then provide patterns of size 1 up to $w$. For temporal transactions, this restricts the events to be bounded within a maximal time interval. An ambiguous pattern contains at least one event that present a given degree of polymorphism. Let suppose that an event A may be substituted for another event $\mathrm{B}$ and that $w=4$, then the sequences CEAD and CBFD are two possible occurrences of the same pattern CAD. A better representation of this pattern is $\mathrm{C}[\mathrm{A} \mathrm{B}] \mathrm{D}$; this notation denotes the interchangeable events inside a pair of brackets.

In this paper we shortly describe the original Winepi algorithm proposed by Toivonen and Mannila [1], then we introduce the concept of ambiguous event as well as the definition of substitution costs, We next show how it can be implemented into the Winepi algorithm. Finally one presents an application to bioinformatics : the discovery of conserved patterns in a family of protein sequences, namely the cytokine receptors.

\section{Data mining algorithms for sequence analysis}

The AprioriAll algorithm [2] finds all the patterns having a minimal support, but presents some inconvenients such as an expensive data transformation and the absence of timing window. GSP [3] is an improvment of AprioriAll that also generalizes the concept of item by using different levels of a user-defined taxonomy. These algorithms are based on the generation of candidate patterns and the counting of these candidates to check their actual frequency. Similar techniques have been used by Mannila and Toivonen [1], [4]. We propose in this paper an adaptation of Winepi [1] to the discovery of ambiguous patterns.

\section{Principle of Winepi}

An event $\mathrm{E}$ is a pair $(A, t)$ where $A \in \Omega$ is an event type and $t$ is an integer. The index $t$ may denote the temporal occurrence of the event $\mathrm{E}$ in a sequence $s$, or simply its position. An episode $\alpha=\left(A_{1}, t_{1}\right),\left(A_{2}, t_{2}\right) \ldots\left(A_{k}, t_{k}\right)$ is an event sequence ordered by the time (i.e. $t_{1} \leq t_{2} \leq \ldots \leq t_{k}$ ) such as $\left|t_{k}-t_{1}\right| \leq w$, where $w$ is the length of the timing window. As we do not care of the occurrence $t_{i}$ of each individual event in $\alpha$, we will only define the episode by the succession of its events, i.e. $\alpha=A_{1} \ldots A_{k}$. A pattern represents a frequent episode ; the frequency (or support) of the pattern is the proportion of sequences that contain this pattern. THe frequency of a pattern must be greater than an input parameter minFreq.

The basic structure of Winepi comprises two key aspects ; the generation of potentially frequent episodes, called candidate episodes, and the actual counting of these candidates. The algorithm makes several passes over the data, at each 
pass, it increases the size $k$ of the episodes. Winepi starts with a size $k=1$ and considers that each individual event belonging to an alphabet $\Omega$ is a potential pattern. Then $k$ is increased of one unit at each pass. The algorithm terminates when there are no frequent sequences at the end of a pass or when there are no candidate episodes generated :

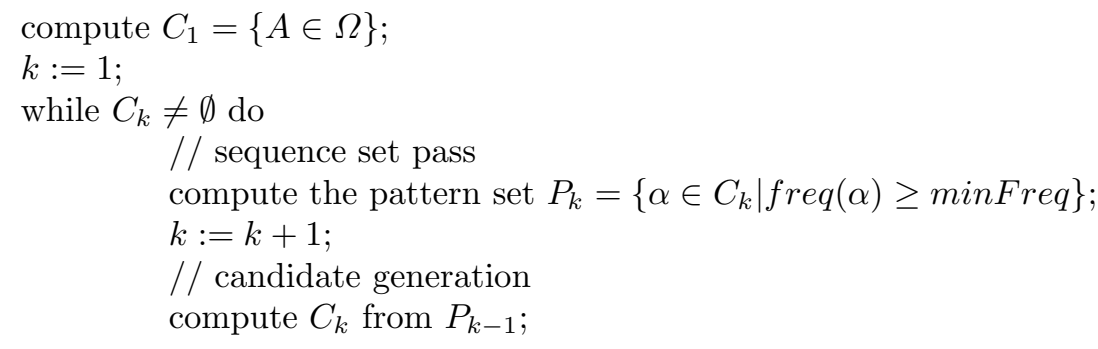

\subsection{Generation of episodes}

An episode is an ordered sequence of events that is generated by the joining of two subepisodes. A subepisode $\alpha^{\prime}$ of size $k^{\prime} \leq k$ contains $k^{\prime}$ events belonging to the episode $\alpha$ and occurring in the same order (e.g. ABC is a subepisode of ADBEF but not ACB). The candidate generation phase is based on a joining procedure that takes two patterns from the pattern set $P_{k-1}$ to form a new episode $\alpha$ of size $k$. A pattern $p_{1}$ joins with a pattern $p_{2}$ if the subepisode obtained by dropping the first event of $p_{1}$ is the same as the subepisode obtained by dropping the last event of $p_{2}$ : the episode $\alpha=A_{1} \ldots A_{k}$ is a new candidate if there exist in $P_{k-1}$ two subepisodes verifying $p_{1}=A_{1}^{1} \ldots A_{k-1} 1^{1}, p_{2}=A_{2}^{2} \ldots A_{k}^{2}$ such as $A_{i}^{1}=A_{i}^{2}$ $\forall i \in[2, k-1]$.

The joining procedure provides a set of potential candidates that is next checked by a pruning procedure. This latter removes all the candidates that cannot be frequent; the pruning is based on the fact that all the subepisodes of a frequent episode must be frequent. Therefore, if there exist at most one subepisode that does not have a minimum support, the potential episode created by the joining procedure will not be inserted in $C_{k}$.

\subsection{Computation of the frequency of the episodes}

During the second phase, the algorithm counts the occurrences of the elements of $C_{k}$ in the sequence set. The algorithm performs this computation in a single pass and takes advantage of the sliding window by recognizing episodes through incremental updates. Winepi is based on a set $\mathcal{A}$ of state automatum that accept candidate episodes and ignore all other input. When a new event $\mathrm{E}$ enters in the window, an automaton $a$ will be initialize for all the episodes begining with $\mathrm{E}$ and will be added to $\mathcal{A}$. When the same event $\mathrm{E}$ leaves the window, $a$ is removed from the set $A$. An efficient data structure stores $\mathcal{A}$ and handles the corresponding state transitions. 


\subsection{Ambiguous events}

In Winepi, the concept of frequency (or support) of an episode $\alpha$ relies on a counting of the observed occurrences of $\alpha$ in the database. The introduction of the concept of ambiguous event necessitates to modify this notion of frequency. We propose a scoring scheme that is commonly used in different fields and especially in bioinformatics : substitution matrices [5]. A substitution matrix $\mathcal{M}$ expresses the cost $w(i, j)$ associated to the substitution of the event $\mathrm{i}$ by the event $\mathrm{j}$. We denote compatible $(X)$ the set of events that can be substituted for $X: \operatorname{compatible}(X)=\{Y \in \Gamma \mid w(X, Y)>0\}$.

We propose to generalize the original concept of frequency that has been developed in Winepi. An exact occurrence of an episode ABC will conserve a score of 1 while ambiguous occurrences will have a lower score. Let consider the sequence $s=\left(s_{1} s_{2} \ldots s k\right)$ as a potential occurrence of a given episode $e=$ $\left(e_{1} e_{2} \ldots e_{k}\right)$. We then define a scoring function $f(s, e)$ as follows :

$$
f(s, e)=\frac{1}{k} \sum_{i=1}^{k} w\left(s_{i}, e_{i}\right) / w\left(e_{i}, e_{i}\right)
$$

where $\mathrm{w}(\mathrm{a}, \mathrm{b})$ are the coefficients of $\mathcal{M}$. Let consider the event alphabet $\Omega=$ $\{A, B, C\}$ and the substitution matrix $\mathcal{M}$ given in Table 1 . In this example, we

Table 1. Substitution matrix

$$
\begin{array}{|r|r|r|r|}
\hline & \mathrm{A} & \mathrm{B} & \mathrm{C} \\
\hline \mathrm{A} & +4 & +2 & -1 \\
\mathrm{~B} & +2 & +5 & -3 \\
\mathrm{C} & -1 & -3 & +7 \\
\hline
\end{array}
$$

have $\mathrm{f}(\mathrm{ABC}, \mathrm{ABC})=1, \mathrm{f}(\mathrm{BBC}, \mathrm{ABC})=0.83, \mathrm{f}(\mathrm{CCC}, \mathrm{ABC})=0.05$. In practise, we will reject incompatible events ; an occurrence of an event $\mathrm{X}$ will be considered as an admissible substitution of the event $\mathrm{Y}$ only if it belongs to compatible $(Y)$. Before a formal presentation of our novel algorithm, let consider on a simple example how we associate a current score to each episode. Let suppose that the window size $w$ is 4 , that the observed sequence is $\mathrm{ABCA}$ and that the concerned episode $\alpha$ is AA. The first event A enters the window and creates an new instance of an automaton $a_{1}$ associated to $\alpha$, since this input corresponds to the first event of $\alpha$. The partial score is $w(\mathrm{~A}, \mathrm{~A}) / w(\mathrm{~A}, \mathrm{~A})=1$. The next event $\mathrm{B}$ is considered as a substitution of $\mathrm{A}$ and the automaton writes that it reaches its second position with a score of $w(\mathrm{~B}, \mathrm{~A}) / w(\mathrm{~A}, \mathrm{~A})=0.5$. The third event $\mathrm{C}$ is neglicted since it does not belong to compatible(A). The algorithm next considers the last event A and notices that it corresponds to the second position of $\alpha$ with a better score : the last score is then overwritten. As it also corresponds to a first position of $\alpha$, a new instance $a_{2}$ of automaton is created as well. Finally the first event leaving 
the window involves the removal of the automaton $a_{1}$. This latter reaches its last state, which means that $\alpha$ has been detected, with a global score of $(1+1) / 2=1$.

The Winepi algorithm is rather sophisticated and for concision sake, we will not give its detailed presentation ; it uses different data structures that optimize the management of $\mathcal{A}$, the interested reader can refer to [1] for further details. We will only stress on the major modifications that we made, concerning the ambiguous events. We have maintained the principle of a list waits that contains all the episodes that are waiting for their $i t h$ element. For instance, the event $\mathrm{A}$ is waited by the episode $\gamma=\mathrm{AC}$ on its first position and by $\beta=\mathrm{BA}$ on its second position, if one assumes that the event $\mathrm{B}$ has already been met. The corresponding lists are : A.waits $[1]=\gamma$ and A.waits $[2]=\beta$. The main difference with the original version of Winepi lies in the fact that a new event $Y$ entering in the window is now considered as a potential ambiguous event and is replaced by each event $X$ belonging to compatible $(Y)$.

The computation of the frequency is also replaced by scores that are dynamically updated for each new event $X$. We need to associate to a current episode $\alpha$ an array of partial scores $\alpha . s$. The size of this array is $k$ and $\alpha . s[i]$ is the current score of the episode $\alpha[1] \ldots \alpha[i]$. We initialise $\alpha . s[i]=0 \forall i \in[0 . . k]$ (for convenience, we add an extra score $\alpha . s[0]$ that always remains 0$)$. We give below the algorithm that takes into account an input event $Y$.

$$
\begin{aligned}
& \text { for } i:=k \text { downto } 1 \text { do } \\
& \qquad \begin{array}{r}
\text { for all } X \in \operatorname{compatible}(Y) \text { do } \\
\text { for all } \alpha \in X . w a i t s[i] \text { do } \\
\qquad \alpha . s[i]:=\alpha . s[i-1]+f(X, \alpha[i]) ;
\end{array}
\end{aligned}
$$

Note that by definition all episodes are always waiting for their first event. The frequency of an episode is the sum of the best scores obtained on each sequence, divided by the number of sequences.

\section{Discovery of conserved patterns in protein sequences}

Knowledge discovery is a promising new research area in bioinformatics [6]. We propose an application to the identification of conserved patterns in a family of proteins. Proteins are linear macromolecules that are assembled from twenty different amino acids hooked together in long chains by chemical bonds. These amino acids are coded by a standard alphabet (e.g L states for Lysine), so that the protein can be represented as a simple string of characters. Cytokines are soluble proteins that are involved in various aspect of cellular proliferation and differentiation, especially in hematopoiesis and immune reactions. Their cell surface receptors are composed of the non-covalent association of two or three transmembrane receptor subunits. These subunits display conserved structural features indicating that they have evolved from a common ancestor. The receptor family that we use comprises 31 sequences of various lengths, from 229 up to 1165 
residues. The overall signature of this family is rather complex, so we will focus only on a particular region that is especially characteristic for which the following patterns have been found : G-W-S-E-W-S-P, score $=0.827$ and G-W-S-D-W-S-P score $=0.821$. A refinement phase should be added to our tool to obtain the PROSITE-like pattern G-W-S- [E D $]-W-S-P$.

\section{Conclusion and future works}

The concept of ambiguous event permits to consider polymorphic data and our novel definition of the frequency defines the degree of transformation that is acceptable for an ambiguous pattern. We show that sequence analysis algorithms can be adapted to take into account this new paradigm ; we successfully apply our scoring scheme to the Winepi algorithm. No doubt that knowledge discovery will be a major issue in computational biology, as it has been predicted by many authors [6]. Our first results show that our algorithm is robust and efficient, but that the presentation of the results must be improved. We are currently working on the adaptation of the Minepi algorithm [4] so that it accepts ambiguous events. Minepi is an alternative method for the discovery of patterns proposed by the authors of Winepi. The Minepi algorithm is more powerful than Winepi, in the sense that it only treats the actual occurrences of the patterns in the database. This efficiency has a conterpart : Minepi is more memory expensive than Winepi. But we are confident that this algorithm may be applied to the case of protein sequences.

\section{References}

1. H. Mannila, H. Toivonen, and AI Verkamo. Discovering frequent episodes in sequences. In First International Conference on Knowledge Discovery and Data Mining (KDD'95), pages 210 - 215. AAAI Press, August 1995.

2. R. Agrawal and R. Srikant. Mining sequential patterns. In 11th International Conference on Data Engineering, March 1995.

3. Ramakrishnan Srikant and Rakesh Agrawal. Mining sequential patterns: Generalizations and performance improvements. In Peter M. G. Apers, Mokrane Bouzeghoub, and Georges Gardarin, editors, Proc. 5th Int. Conf. Extending Database Technology, EDBT, volume 1057 of Lecture Notes in Computer Science, LNCS, pages 3-17. Springer-Verlag, 25-29 March 1996.

4. Heikki Mannila and Hannu Toivonen. Discovering generalized episodes using minimal occurrences. In 2nd International Conference on Knowledge Discovery and Data Mining (KDD'96), pages 146 - 151. AAAI Press, August 1996.

5. S Henikoff and JG Henikoff. Amino acid substitution matrices from protein blocks. Proc. Natl. Acad. Sci. USA, 89:10915-10919, 1992.

6. Janice Glasgow, Igor Jurisica, and Raymond Ng. Data mining and knowledge discovery in databases. In Pacific Symposium on Biocomputing (PSB '99), January 1999. 